\title{
Bicomponent droplets evaporation: Temperature measurements and modelling
}

\author{
C. Maqua, G. Castanet, F. Lemoine * \\ LEMTA, Nancy-Université, CNRS 2, Avenue de la forêt de Haye, BP 160, F-54504 Vandoeuvre-lès-Nancy, France
}

\begin{abstract}
A B S T R A C T
Development of improved energy conversion systems, having higher efficiencies and lower emissions, is central to reducing the production of green-house gases and to meeting air quality standards for other emissions. In non-premixed combustion systems, vaporization of the droplets and mixing of the vapour with the surrounding oxidizer control the overall rate of energy release. Droplet vaporization is affected by the nature of the liquid petroleum that contains species having extremely different volatilities. A fine understanding of multi-component droplet vaporization is therefore an important issue to optimize the combustion systems. This paper presents the application of a recently developed technique to measure the temperature of bi-component droplets. Based on the three-color laser-induced fluorescence (LIF) technique, droplet temperatures can be measured regardless of the composition. The method requires adding a small amount of a fluorescent organic dye to the fuel which is composed of ethyl-alcohol and acetone. The accuracy of the measurement is about $\pm 1.3^{\circ} \mathrm{C}$. In this study, the emphasis is placed on the evaporation of the binary mixture in a hot air plume, the temperature of which (around $650{ }^{\circ} \mathrm{C}$ ) largely exceeds the boiling point of the liquid components. An extensive study of the influence of the initial composition and droplet diameter is carried out. Finally, the experimental results are compared to an evaporation model based on the discrete components approach.
\end{abstract}

Keywords:

Multicomponent droplets

Temperature

Evaporation activity in the liquid phase $[3,4]$. The description is completed by an appropriate modelling of the heat and mass transfers within the droplets. Predictions can be achieved by the diffusion limit model (DL). In this approach, transfers in the droplet are radial $[1,5]$. This type of model was used by Landis and Mills [2] who investigated the vaporization of bicomponent fuel droplets in a stagnant atmosphere. A more refined model, which accounts for the internal circulations within the droplets due to the gas friction at the droplet surface, was developed by Prakash and Sirignano $[6,7]$. The streamlines are approximated by the Hill vortex solution. This model was adapted to simulate the evaporation of multi-component droplets [8]. Abramzon and Sirignano [9] suggested including the effects of internal circulation through an effective diffusivity making the resolution as simple as with the DL model.

Nevertheless, both DL and Hill vortex models are unpractical when dealing with fuels that contain hundreds of chemical species due to an exponentially growing computing time. Possible alternatives include the continuous thermodynamics [10] and the distillation curve models [11] which both assumed that the temperature and the concentration of the chemical species are spatially uniform but time varying in the droplet. This assumption may be reliable when the droplet lifetime is much longer than the time required to transport the temperature and the concentration from the droplet surface to its core. However, it is particularly questionable when the droplet evaporation proceeds at temperatures well above the boiling point of the fuel.
* Corresponding author. Tel: + 333835957 32; fax: +33 383595544 E-mail address: fabrice.lemoine@ensem.inpl-nancy.fr (F. Lemoine). 


\begin{tabular}{|c|c|c|c|}
\hline \multicolumn{4}{|c|}{ Nomenclature } \\
\hline$a$ & thermal diffusivity & $\eta$ & droplet to droplet interaction factor \\
\hline$B_{\mathrm{M}}$ & Spalding mass transfer number & $\mu$ & dynamic viscosity \\
\hline$B_{\mathrm{T}}$ & Spalding heat transfer number & $v$ & kinematic viscosity \\
\hline$C_{p}$ & specific heat capacity & $\lambda$ & thermal conductivity \\
\hline$C^{p}$ & $\begin{array}{l}\text { non-dimensional distance parameter, concentration of } \\
\text { the fluorescence dye }\end{array}$ & $\chi$ & $\begin{array}{l}\text { molar fraction } \\
\text { specific density }\end{array}$ \\
\hline$D$ & droplet diameter or $D_{i}$ diffusivity of the $i$ th specie & $\Phi$ & heat flux \\
\hline$I$ & fluorescence intensity & $\psi$ & stream function \\
\hline$L_{\mathrm{v}}$ & latent heat of vaporization & & \\
\hline$m$ & droplet mass & \multicolumn{2}{|c|}{ Subscripts } \\
\hline$n$ & mole number & $\mathrm{g}$ & gas phase \\
\hline $\mathrm{Nu}$ & Nusselt number & $i$ & $i$ th chemical specie in the mixture \\
\hline$P$ & pressure & iso & isolated droplet \\
\hline$R$ & droplet radius or ideal gas constant & inj & injection \\
\hline Re & droplet Reynolds number & 1 & liquid phase \\
\hline$R a$ & Rayleigh number & ref & reference \\
\hline Sh & Sherwood number & $S$ & droplet surface \\
\hline$T$ & temperature & sat & saturation condition \\
\hline$U_{\mathrm{s}}$ & maximum velocity at the droplet surface & 0 & initial condition \\
\hline$V$ & velocity & $\infty$ & far field condition \\
\hline Y & mass fraction & & \\
\hline Z & volume fraction of ethanol & & \\
\hline \multicolumn{4}{|c|}{ Greek symbols } \\
\hline$\beta$ & volumetric thermal expansion coefficient & & \\
\hline$\gamma$ & chemical activity & & \\
\hline
\end{tabular}

Presently, few experimental data are available on the thermal evolution of multi-component droplets that evaporates in hot gas environments. Computational models are commonly validated by measured droplet sizes and velocities, while temperature is generally missing due to the lack of measurement techniques available to handle the case of multicomponent droplets. However the characterization of the droplet temperature would be all the more interesting that the droplet heat up period can generally not be neglected in many industrial applications. This paper presents the application of a recently developed technique based on the three-color laser-induced fluorescence. In a previous study [12], the technique was demonstrated on binary droplets made of acetone and ethanol and results were obtained for droplets evaporating in the vicinity of the ambient temperature.

In the present study, monodisperse droplet streams are injected into a hot air plume to investigate the effects of hot gas conditions and a parametric study of the influence of the initial composition and droplet size is presented. Experimental data are then compared to a model that uses the discrete component approach.

\section{Experimental facilities}

\subsection{Droplets generation and experimental conditions}

Many studies concerning droplet evaporation and combustion were dealing with linear monodisperse droplet streams [15]. They consist on a line of droplets injected repeatedly at a well-controlled frequency, size and velocity. The effect of each of these parameters on the vaporization rate can be characterized separately which makes this configuration particularly attractive. For instance, experiments can be useful to validate CFD codes on idealized cases where all the flow parameters are well defined.

A monodisperse droplet stream is generated by Rayleigh-type disintegration of a liquid jet using a piezoceramic [16]. The fuel temperature is regulated in the injector body and is measured near the outlet by a thermocouple. Downstream distance from the injection point is converted into time with the help of the evolution of the droplet velocity that is determined by Laser Doppler Anemometry (LDA). Droplets are injected either into a quiescent atmosphere at ambient temperature or into a hot co-flowing air stream, released from two electrical heaters, arranged symmetrically relative to the droplet streaming axis (Fig. 1). The air is ejected at about $2 \mathrm{~m} / \mathrm{s}$ as measured by LDA and the profile of the air velocity along the plume axis is plotted in Fig. 1. The temperature of the air flow, measured by a thermocouple, varies from $550{ }^{\circ} \mathrm{C}$ to about $100{ }^{\circ} \mathrm{C}$ at $6 \mathrm{~cm}$ from the injection point.

\subsection{Measurement system}

A comprehensive survey of the two-colours laser-induced fluorescence applied to the temperature measurement of single component droplets can be found in $[13,14]$. It has been extended by a third detection band as described by Maqua et al. [12] to address the case of binary droplets. The technique requires the seeding of the fuel by a low concentration of a temperature sensitive fluorescent dye (rhodamine $B$ in the present case). The fluorescence of rhodamine $B$ is induced by the green line of the argon ion laser $(\lambda=514.5 \mathrm{~nm})$ and its intensity is then detected on three separate spectral bands. An important feature for the measurement of the droplet temperature is that the ratios of the fluorescence intensities of these bands are independent on the dimensions of the detection volume, the local laser intensity and the tracer concentration and only depends on the temperature and the composition of the droplet. Additionally, a reference measurement is required to infer the temperature of the droplets from the intensity ratios. This reference is performed at ambient temperature (i.e. with the hot air generator turned off), on the liquid cylindrical jet before its disintegration into droplets. In these conditions, the temperature can be safely assumed to correspond to the temperature measured in the injector by means of the thermocouple and the 

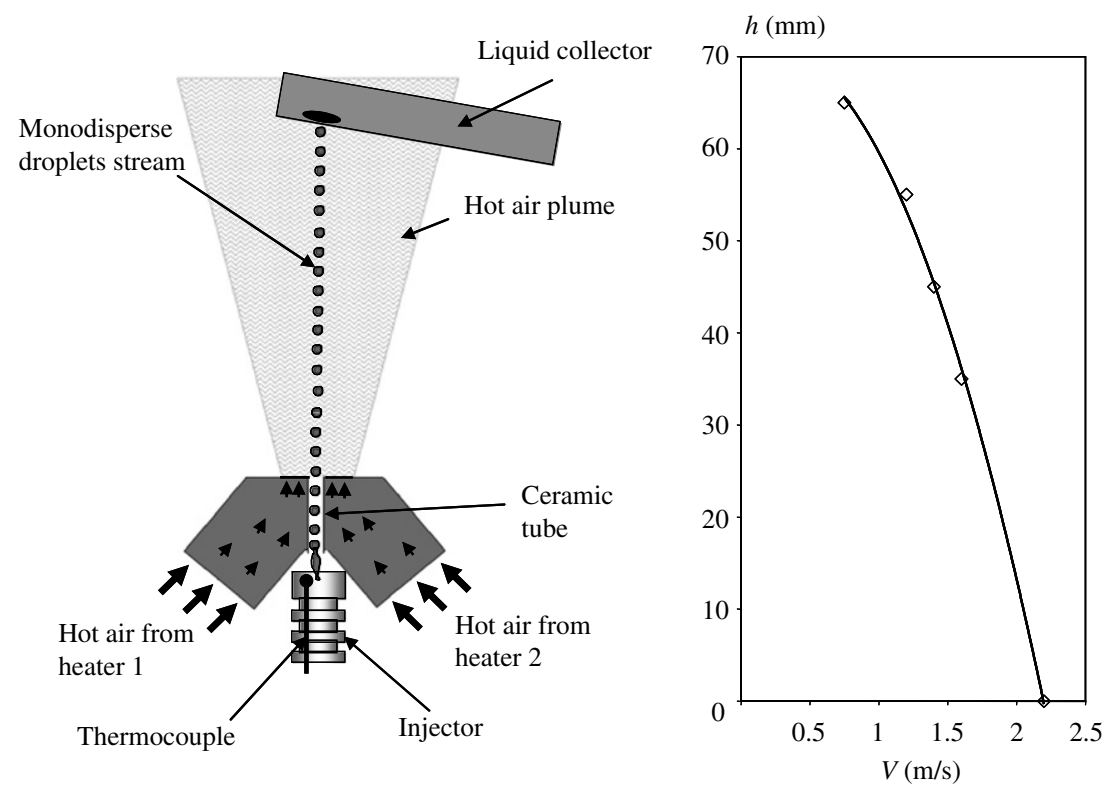

Fig. 1. Schematic view of the droplet injection into the hot air plume and air velocity profile along the streaming axis.

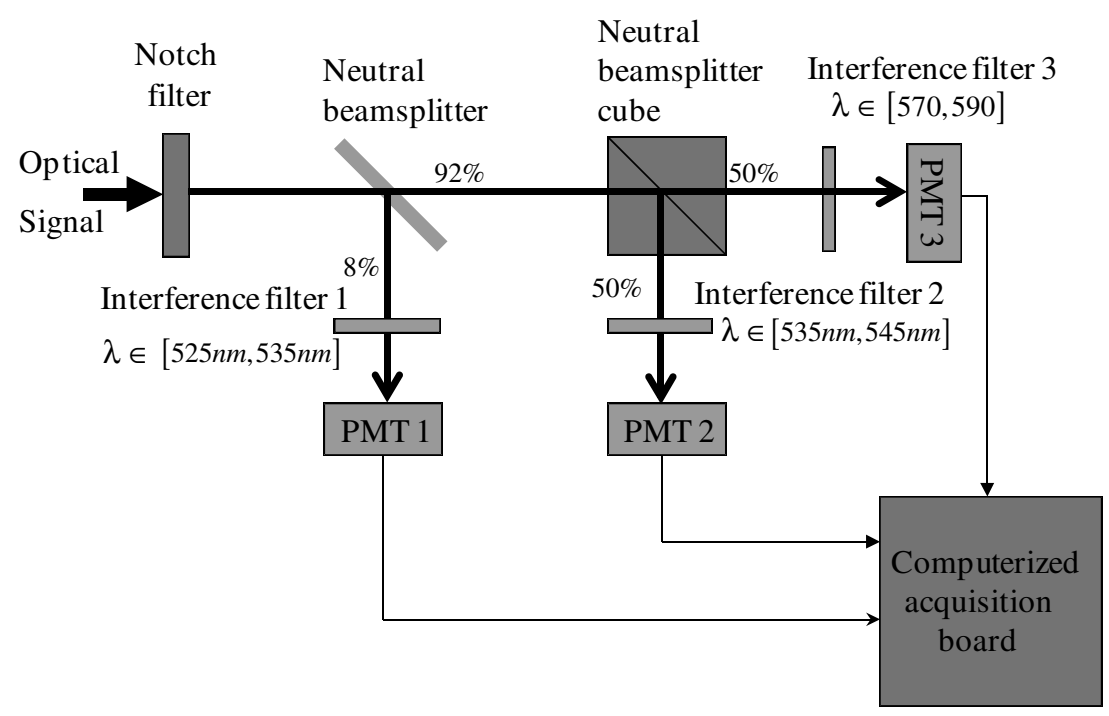

Fig. 2. Block diagram of the three colours LIF experimental set-up.

ethanol volume fraction of the liquid is the same as in the injector. Two laser beams are focused into the LDA measuring volume, which is also the probe volume of the fluorescence measurements. The fluorescence signal is detected at right angle by an achromatic doublet connected to an optical fiber. The laser light $(\lambda=514.5 \mathrm{~nm})$ coming from the droplets is eliminated by a notch filter in order to detect only the fluorescence emission. The fluorescence signal is then divided in the three spectral bands by a set of beamsplitters and interference filters (Fig. 2). The detection is performed by means of three photomultipliers. Finally, the signal processing is performed by a multi-channel acquisition board with a sampling rate of $5 \mathrm{MHz}$. The accuracy of the temperature measurement is about $1.3^{\circ} \mathrm{C}$. However the characterization of the ethanol volume fraction is less accurate and estimated at about $4 \%$.

At this stage, it should be added that the evolution of the droplet sizes is not measured, but the initial diameter is determined by a measurement of the fuel flowrate $Q$ at the injector exit. The droplet size $D_{0}$ is then inferred from:
$D_{0}=(6 Q / \pi f)^{1 / 3}$

where $f$ denotes the piezoceramic frequency.

\section{Physical modeling and simulations}

\subsection{Modelling of the heat and mass transfers}

The modelling of the droplet evaporation considers the case of a droplet that maintains a perfectly spherical shape despite the aerodynamics stress and possible Marengoni effect. The heat transfer inside the liquid droplets results from pure heat conduction and advection by the internal motions due to the friction between the liquid droplet surface and the external air flow. The evolution of the liquid composition can be described by the discrete components approach presented by Sirignano [17], which consists in following each chemical species individually. Taking into account the axisymmetry of the problem, the mass fraction of the $i$ th species $Y_{1, i}$ 

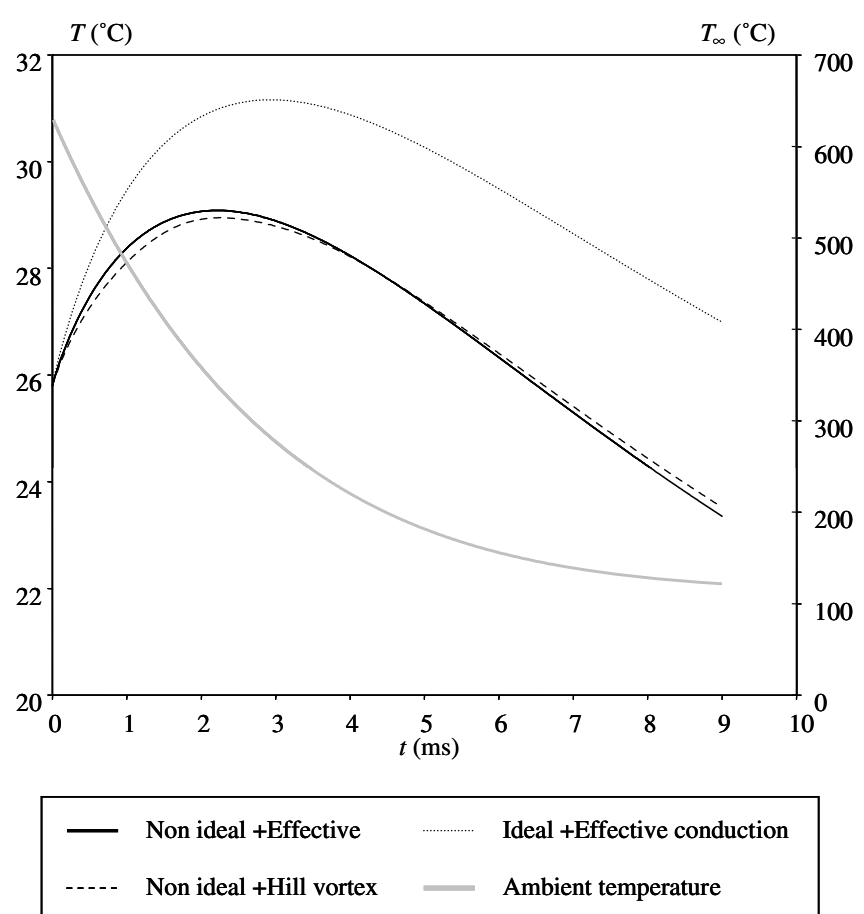

Fig. 3. Calculated evolution of the droplet temperature for different modelling assumptions Conditions in the hot air plume, boundary temperature represented on the secondary axis $\left(D_{0}=130 \mu \mathrm{m}\right.$ and $\left.Z_{0}=0.5\right)$.

and the temperature $T$ within the droplet are derived from the following equation:

$\frac{\partial \xi}{\partial t}-\frac{r^{*}}{R} \frac{\mathrm{d} R}{\mathrm{~d} t} \frac{\partial \xi}{\partial r^{*}}+\frac{1}{R}\left(v \cdot \nabla_{r^{*}}\right) \xi=\frac{D_{\xi}}{R^{2}} \Delta_{r^{*}} \xi$

$\xi$ denotes either the temperature $T$ or the mass fraction $Y_{i}$. The equation is written in the spherical coordinates where $r$ corresponds to the radial distance to the centre of the droplet. $r^{*}$ is the normalised radius coordinate $r^{*}=r / R$. The evolution of the droplet radius $R$ has to be derived separately by integrating the droplet evaporation rate $\dot{m}=\sum_{i} \dot{m}_{i}$ over the time. $v$ is the velocity field within the droplet.

A rather accurate modelling approach lies in the assumption that the internal streamlines of the liquid circulating within the droplet follows a spherical Hill vortex pattern. The stream function is deduced from the Hill vortex solution [18].

$\psi\left(r^{*}, \theta, \phi\right)=-\frac{U_{\mathrm{S}} R^{2}}{2} r^{* 2}\left(1-r^{* 2}\right) \sin ^{2} \theta$

where $\left(r^{*}, \theta, \phi\right)$ are the spherical coordinates of a point within the droplet. This flow pattern is expected to be valid for a wide range of Reynolds number as explained by Sirignano [17]. Only the maximum surface velocity $U_{\mathrm{S}}$ should change significantly depending on the conditions (relative viscosities, Stefan flow, ...). From the shear stresses balance at the droplet surface, the maximum surface velocity $U_{\mathrm{S}}$ can be estimated as suggested in [9]:

$U_{\mathrm{s}}=\frac{1}{6 \pi} \frac{\mu_{\mathrm{g}}}{\mu_{\mathrm{l}}}\left(V-V_{\infty}\right) \operatorname{ReC}_{F}$

In this expression, $C_{F}=\frac{12.69}{R e^{2 / 3}\left(1+B_{M}\right)}$ is the friction coefficient, $R e$ is the droplet Reynolds number based on the droplet diameter and $B_{\mathrm{M}}$ is the Spalding mass transfer number defined as $B_{\mathrm{M}}=\frac{\sum_{i} Y_{i, S}-\sum_{i} Y_{i, \infty}}{1-\sum_{i} Y_{i, S}}$. The term $\left(1+B_{\mathrm{M}}\right)$ is introduced here to consider the reduction of the friction coefficient induced by the Stefan flow. In the gas phase, heat and mass transfers are considered as quasi-steady and the thermophysical properties are assumed to be spatially uniform but time varying. These simplifications can lead to correct results provided that the physical properties are calculated at a reference state. Hubbard et al. [19] recommended the use of the "1/3 rule":

$T_{\text {ref }}=T_{\mathrm{s}}+\left(T_{\infty}-T_{\mathrm{s}}\right) / 3$ and $Y_{i, \text { ref }}=Y_{i, \mathrm{~S}}+\left(Y_{i, \infty}-Y_{i, \mathrm{~S}}\right) / 3$

The fuel vapour fraction $Y_{i, S}$ is determined assuming a liquid-vapour equilibrium:

$\chi_{i, \mathrm{~g}, \mathrm{~S}} P_{a}=\chi_{i, \mathrm{I}, \mathrm{S}} \gamma_{i} P_{\mathrm{sat}}\left(T_{\mathrm{S}}\right)$

where $\chi_{i}$ and $\gamma_{i}$ are, respectively, the molar fraction and the activity coefficient in the liquid phase related to the $i$ th component of the mixture. Since experiments will be carried out at atmospheric pressure, the gas phase can be safely considered as an ideal mixture. In the liquid, the activity coefficients $\gamma_{i}$ can be calculated from the total excess Gibbs energy $G^{\mathrm{E}}$ :

$R T \ln \left(\gamma_{i}\right)=\left(\frac{\partial\left(n_{1} G^{\mathrm{E}}\right)}{\partial n_{j, 1}}\right)_{T, P, n_{j \neq i}}$

where $n_{1}$ is the mole density (moles per unit volume) in the liquid mixture and $n_{i, 1}$ denotes the mole density of the component $i$. An
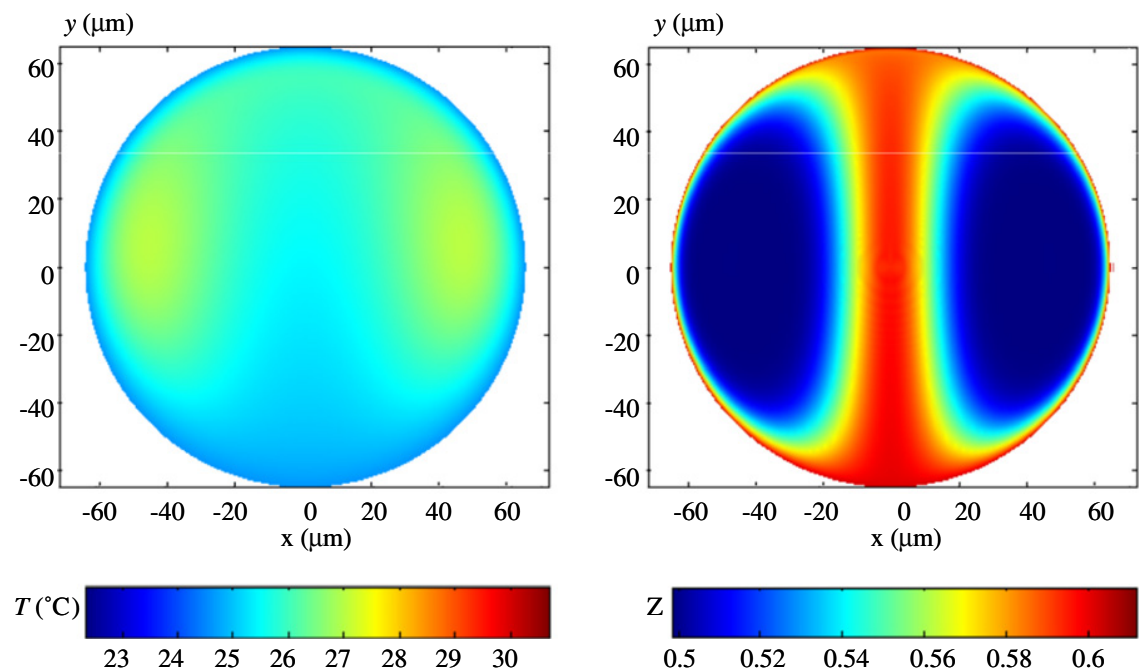

Fig. 4. Calculated equatorial maps of temperature and ethanol volume fraction in the hot air plume at $t=6.5 \mathrm{~ms}$ (same conditions as in Fig. 3 ). 
empirical correlation was proposed by Campbell et al. [20] for the estimation of $G^{\mathrm{E}}$ in the case of binary mixtures:

$\frac{n_{1} G^{\mathrm{E}} / R T}{\chi_{i, 1} \chi_{j, 1}}=\alpha \chi_{i, 1}+\beta \chi_{j, 1}-\delta \chi_{i, 1} \chi_{j, 1}, i \neq j$

where $\alpha, \beta, \delta$ vary with the temperature of the mixture. They are determined experimentally in the case of the acetone-ethanol mixture [20].

Eq. (2) is completed by a set of initial conditions: $T(r, t=0)=T_{\text {inj }}$ and $Y_{i}(r, t=0)=Y_{i, \text { inj }}$ and boundary conditions. To take into account the effect of the convective transport caused by the droplet motion relative to the gas, heat and mass transfers are evaluated in the frame of the so-called 'film theory', which implies uniform but time-varying surface conditions in term of temperature and composition ( $T_{\mathrm{S}}$ and $\left.Y_{i, 1, \mathrm{~S}}\right)$. This theory assumes that the resistance to the transfers can be modelled by introducing a fictitious gas film around the droplet. In the case of evaporating moving droplets, Abramzon and Sirignano [9] have described extensively how the presence of the Stefan flow modifies the thickness of this film. They derived widely accepted expressions of Nusselt and Sherwood numbers. At the droplet surface, the heat flux entering into the droplet is denoted $Q_{L}$ and $\dot{m}_{i}$ is the vapour mass flux of the specie $i$.

$2 \pi R^{2} \lambda_{1} \int_{\theta=0}^{\pi}\left(\frac{\partial T}{\partial r}\right)_{r=R} \sin (\theta) \mathrm{d} \theta=Q_{L}(t)$

$\dot{m}_{i}=\dot{m} Y_{i, 1, \mathrm{~S}}-2 \pi R^{2} \rho_{\mathrm{l}} D_{i ; \mathrm{1}} \int_{\theta=0}^{\pi}\left(\frac{\partial Y_{i, 1}}{\partial r}\right)_{r=R} \sin (\theta) \mathrm{d} \theta$

As an alternative to the Hill vortex model, a simpler approach is the effective diffusivity model introduced by Abramzon and Sirignano [9] in the case of the sole heat transfer. Eq. (2) is solved in the absence of the convective term but the diffusion coefficient $D_{\xi}$ is increased by a factor $\chi$ that depends either on the thermal or the mass Peclet number Pe:

$\chi=1.86+0.86 \tanh \left(2.245 \log _{10}\left(\frac{P e}{30}\right)\right)$ and $P e=\frac{2 R U_{s}}{D_{\xi}}$

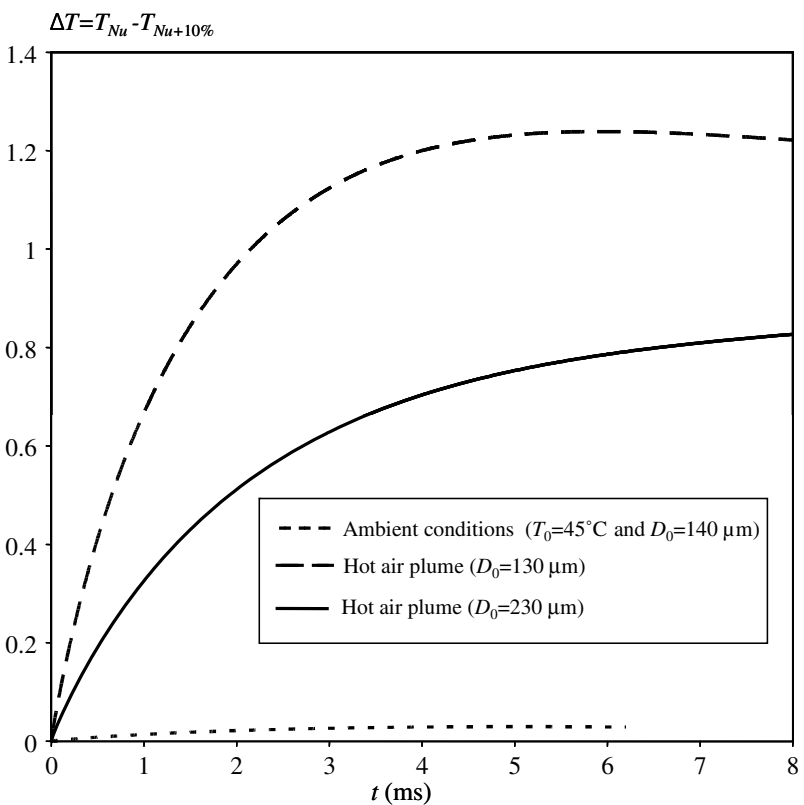

Fig. 5. Evolution of the droplet temperature as a function of the ethanol volume fraction at $t=6.5 \mathrm{~ms}$ (evaporation near the ambient temperature). Comparison to the numerical simulation results for ideal and non ideal mixture hypothesis $\left(D_{0}=140 \mu \mathrm{m}, T_{0}=45^{\circ} \mathrm{C}, T_{\infty}=20^{\circ} \mathrm{C}\right.$, origin of time at the injector exit).
The instantaneous heat flux $Q_{L}$ is derived from the overall energy balance of the evaporation after neglecting the radiation exchanges, which is justified here by the moderate ambient temperature:

$Q_{L}=\Phi_{\mathrm{c}}-\Phi_{\text {vap }}$

where $\Phi_{\mathrm{C}}$ is the convective heat flux transferred from the gas and $\Phi_{\text {vap }}$ is the heat flux due to vaporization. The convective heat flux and the vapour mass flowrate derived from Nusselt and Sherwood numbers are defined by:

$\Phi_{\mathrm{c}}=\pi \lambda_{\mathrm{ref}} D N u\left(T_{\infty}-T_{\mathrm{S}}\right)$

$\dot{m}=\pi \rho_{\text {ref }} D D_{i} B_{\mathrm{M}, i} S h_{i}$

Eq. (14) is valid for any component $i$ contained in the droplet. In the film theory [9], Nusselt and Sherwood numbers of an isolated droplet are given by:

$N u_{\text {iso }}=\frac{\ln \left(1+B_{\mathrm{T}}\right)}{B_{\mathrm{T}}}\left(2+\frac{0.6 R e^{1 / 2} \operatorname{Pr}^{1 / 3}}{F\left(B_{\mathrm{T}}\right)}\right)$

$S h_{i, \text { iso }}=\frac{\ln \left(1+B_{\mathrm{M}, i}\right)}{B_{\mathrm{M}, i}}\left(2+\frac{0.6 R e^{1 / 2} S c_{i}^{1 / 3}}{F\left(B_{\mathrm{M}, i}\right)}\right)$

In the latter equations, $F(B)=(1+B)^{0.7} \frac{\ln (1+B)}{B}$ : $B_{\mathrm{T}}$ is the thermal Spalding number and $B_{\mathrm{M}, i}$ the mass Spalding number

$B_{\mathrm{T}}=\frac{\left(\sum_{i} \dot{m}_{i} C p_{i, \mathrm{~g}}\right)\left(T_{\infty}-T_{\mathrm{S}}\right)}{\left(\sum_{i} \dot{m}_{i} L v_{i}\right)+Q_{L}}$

$B_{\mathrm{M}, i}=\frac{Y_{i, \mathrm{~S}}-Y_{i, \infty}}{\dot{m}_{i} / \dot{m}-Y_{i, S}}$

Dealing with linearly interacting droplets, Castanet et al. [21] proposed to decrease the Nusselt and Sherwood numbers by a

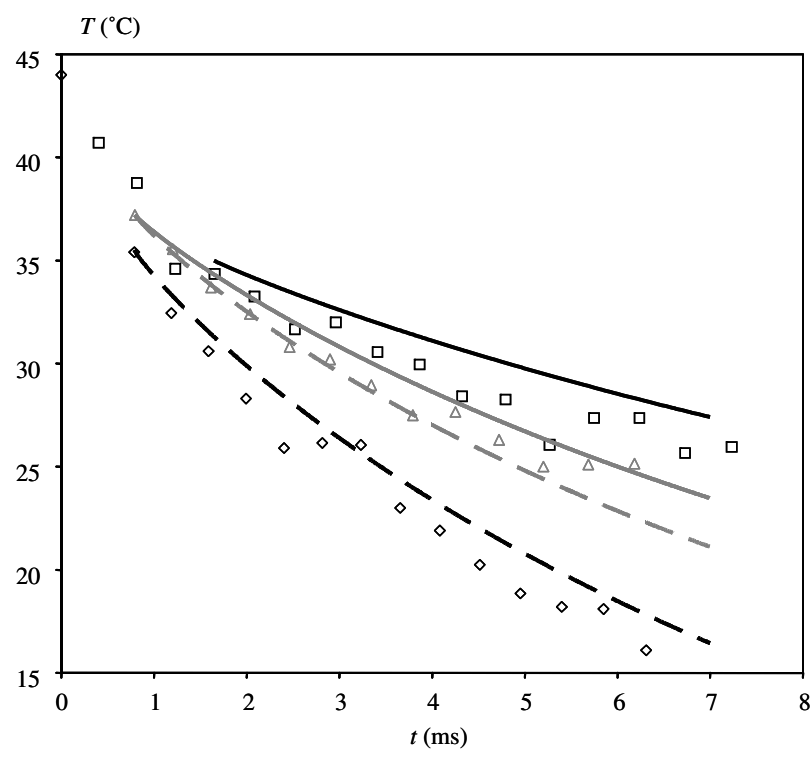

\begin{tabular}{|c|c|c|c|}
\hline Experimental data & Numeric & al results & \\
\hline$\square Z_{0}=1$ & & $\mathrm{Z}_{0}=1$ & \\
\hline$\Delta \mathrm{Z}_{0}=0.5$ & & $\mathrm{Z}_{0}=0.5$ & Ideal mixture \\
\hline$\diamond \mathrm{Z}_{0}=0$ & $\begin{array}{l}-- \\
--\end{array}$ & $\begin{array}{l}\mathrm{Z}_{0}=0.5 \\
\mathrm{Z}_{0}=0\end{array}$ & Non-ideal mixture \\
\hline
\end{tabular}

Fig. 6. Temporal evolution of the droplet temperature for different ethanol volume fraction (evaporation near the ambient temperature). Comparison to the numerical simulation results for ideal and non ideal mixture hypothesis $\left(D_{0}=140 \mu \mathrm{m}, T_{0}=\right.$ $45^{\circ} \mathrm{C}, T_{\infty}=20^{\circ} \mathrm{C}$, origin of time at the injector exit). 
factor $\eta$. They demonstrated that applying an identical value of $\eta$ to Nusselt and Sherwood numbers was sufficient to predict the evolution of the droplet size and temperature of combusting monodisperse streams made of ethanol droplets.

$\eta(C)=\frac{N u}{N u_{\text {iso }}}=\frac{S h}{S h_{\text {iso }}}$

where $C$ is the distance parameter, i.e. the distance between the droplets divided by their diameter.

Atthassit et al. [22] studied the effects of the droplet-to-droplet interaction on the evaporation rate in the case of ethanol droplets evolving into the thermal boundary of vertical heated plate. They proposed a slightly different expression for $\eta$ compared to [21]:

$\eta(C)=1-0.57\left(1-\frac{1-0.57 \mathrm{e}^{-0.13(C-6)}}{1+0.57 \mathrm{e}^{-0.13(C-6)}}\right)$

According to the authors, this expression should be valid in the domain $2.5 \leqslant C \leqslant 16$ and therefore will be used in the present study.

\subsection{Numerical resolution}

The step-by-step resolution procedure has been extensively described by Abramzon and Sirignano [9] in the case of mono-component droplets and the same approach is presently applied. This section gives only a brief overview of the algorithm.

(1) Knowing $T_{\mathrm{S}}$ and $Y_{i, 1, \mathrm{~S}}$ from the initial conditions or the previous time step, the composition of the vapour phase at the droplet surface is determined from Eq. (6) and the ' $1 / 3$ rule' (Eq. (5)) is then used to calculate the properties of the gaseous phase. Dimensionless numbers such as $\mathrm{Re}, \mathrm{Pr}, \mathrm{Sc} c_{i}$ are also estimated. In the case of the Reynolds number, the droplet velocity is required. Although the droplet decelera-

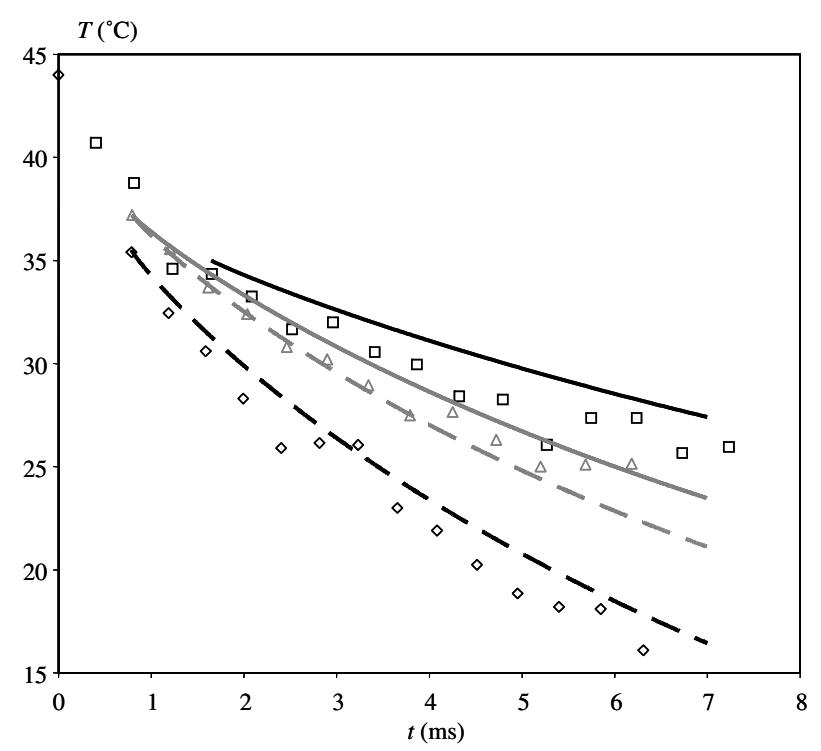

\begin{tabular}{|c|c|c|c|}
\hline Experimental data & Numeric & l results & \\
\hline$\square Z_{0}=1$ & & $\mathrm{Z}_{0}=1$ & \\
\hline$\Delta \mathrm{Z}_{0}=0.5$ & & $\mathrm{Z}_{0}=0.5$ & Ideal mixture \\
\hline \multirow{2}{*}{$\diamond \mathrm{Z}_{0}=0$} & & $\mathrm{Z}_{0}=0.5$ & Non-ideal mixture \\
\hline & & $\mathrm{Z}_{0}=0$ & \\
\hline
\end{tabular}

Fig. 7. Temporal evolution of the droplet temperature for different ethanol volume fraction (evaporation near the ambient temperature). Comparison to the numerical simulation results for ideal and non ideal mixture hypothesis $\left(D_{0}=140 \mu \mathrm{m}, T_{0}=\right.$ $45^{\circ} \mathrm{C}, T_{\infty}=20^{\circ} \mathrm{C}$, origin of time at the injector exit). tion due to the drag force could have been modelled, the droplet velocity is fixed at its experimental value in the simulation. Doing this, any risk of error in the estimation of the drag coefficient is avoided and the attention is focused exclusively on the heating and evaporation processes.

(2) Since the vapour mass flowrate $\dot{m}_{i}$ is included in the mass transfer Spalding number $B_{\mathrm{M} i}$ (Eq. (18)), an iterative procedure should be used to find out the value of $\dot{m}_{i}$ from the Eqs. (14) and (16). As a starting point, $S h_{i}$ is fixed to its value in the previous time step of the resolution. This simplification enables to obtain a first estimate of $\dot{m}_{i}$ after solving Eq. (14). From $\dot{m}_{i}$, an updated value of $S h_{i}$ can be calculated according to Eq. (16). The iterative process is carried on until the convergence error on the value of $\dot{m}_{i} / \dot{m}$ is less than $10^{-6}$.

(3) Using the definition of $B_{\mathrm{T}}$, Eq. (12) can be written in the following form:

$\pi \lambda_{\text {ref }} D N u B_{\mathrm{T}}=\sum_{i} \dot{m}_{i} C p_{i, \mathrm{~g}}$

The latter equation is solved iteratively in order to find out the value of $B_{\mathrm{T}}$ with a convergence error of $10^{-6}$ and $Q_{L}$ is then derived from $B_{\mathrm{T}}$ using Eq. (17).

(4) The temperature and concentration fields within the droplet are calculated with respect to the boundaries conditions (Eqs. (10) and (11)) while maintaining both $T_{\mathrm{S}}$ and $Y_{i, 1, \mathrm{~S}}$ uniform. To solve Eq. (2), the droplet is divided into 100 regular segments along the $r$-coordinate. A time step $\mathrm{d} t$ significantly smaller than the general time step $\Delta t$ is selected so that

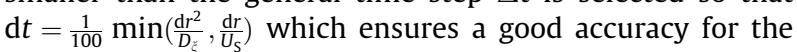
resolution. In the case of the Hill vortex model, $\xi$ is split on
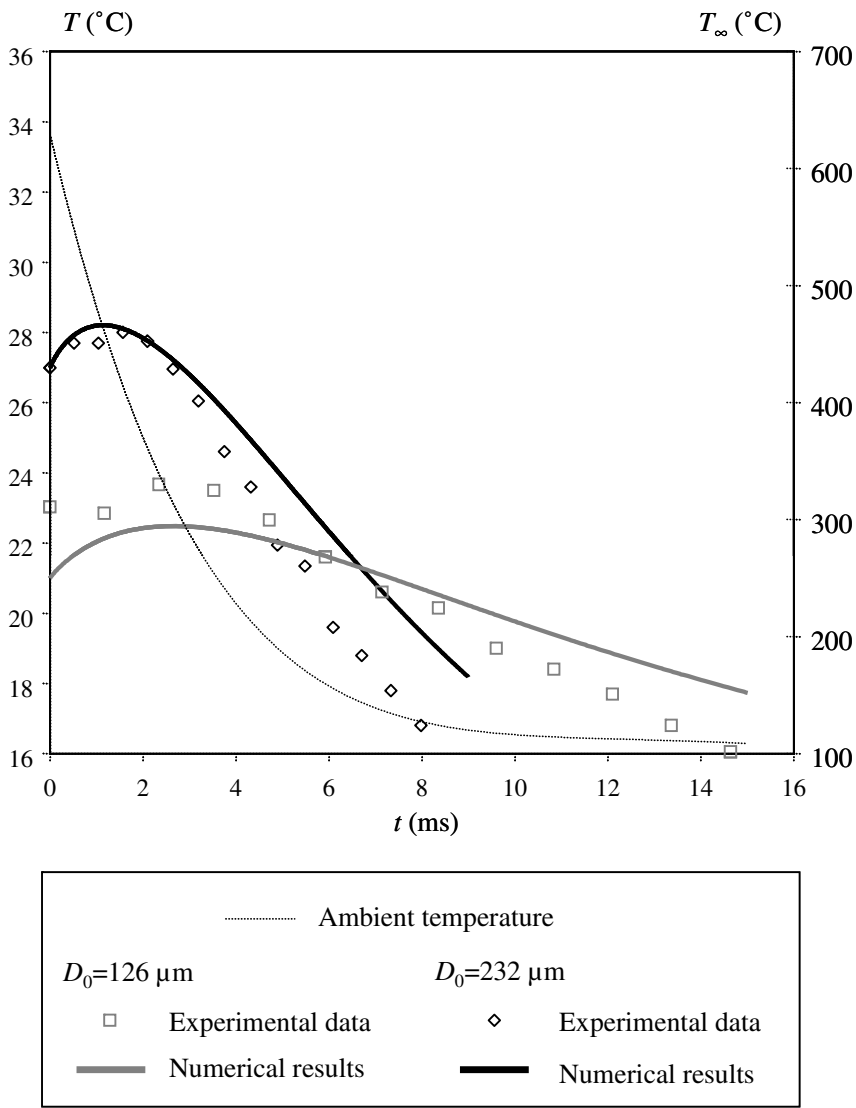

Fig. 8. Temporal evolution of the droplet temperature for two droplet diameters and $Z_{0}=0$ in the hot air plume. Comparison to the numerical simulation results (origin of time at the droplet entrance in the hot air plume). 
the Legendre polynomials base, which enables to remove the dependence on the coordinate $\theta$ [23]. Twenty modes are considered but 10 modes appears sufficient to get reliable results. An explicit second order Adams-Bashfort scheme is adopted for the time integration of Eq. (2). The spatial derivatives are approximated by finites differences with central differences for the second derivatives in the diffusion term of Eq. (2). In the case of the effective diffusion model, a Crank-Nicholson scheme is used exactly as described in [9].

Finally, the values of $T_{\mathrm{S}}$ and $Y_{i, 1, \mathrm{~S}}$ are determined at $t+\Delta t$, and the calculation return to stage 1 . The thermo physical properties, the entering heat flux $Q_{L}$, the vapour mass flowrate $\dot{m}$ are updated with a time step $\Delta t$ of $0.01 \mathrm{~ms}$.

\section{Results}

\subsection{Preliminary tests of the model}

Preliminary tests have been carried out in order to assess the reliability of the heat and mass transfer models, i.e. Hill vortex or effective diffusion. The following initial conditions were used: $D_{0}=130 \mu \mathrm{m}, T_{0}=25.8^{\circ} \mathrm{C}, Z_{0}=0.5$ and $C_{0}=7.3$. The droplets were injected into the hot air flow described in Section 2.1. The time evolution of the droplet velocity is known from the LDA measurements. Droplets that are injected upwards undergo a deceleration. In Fig. 3, numerical simulations clearly show that the effective diffusion and Hill vortex models give similar results. This was expected since the effective diffusion model was developed in the

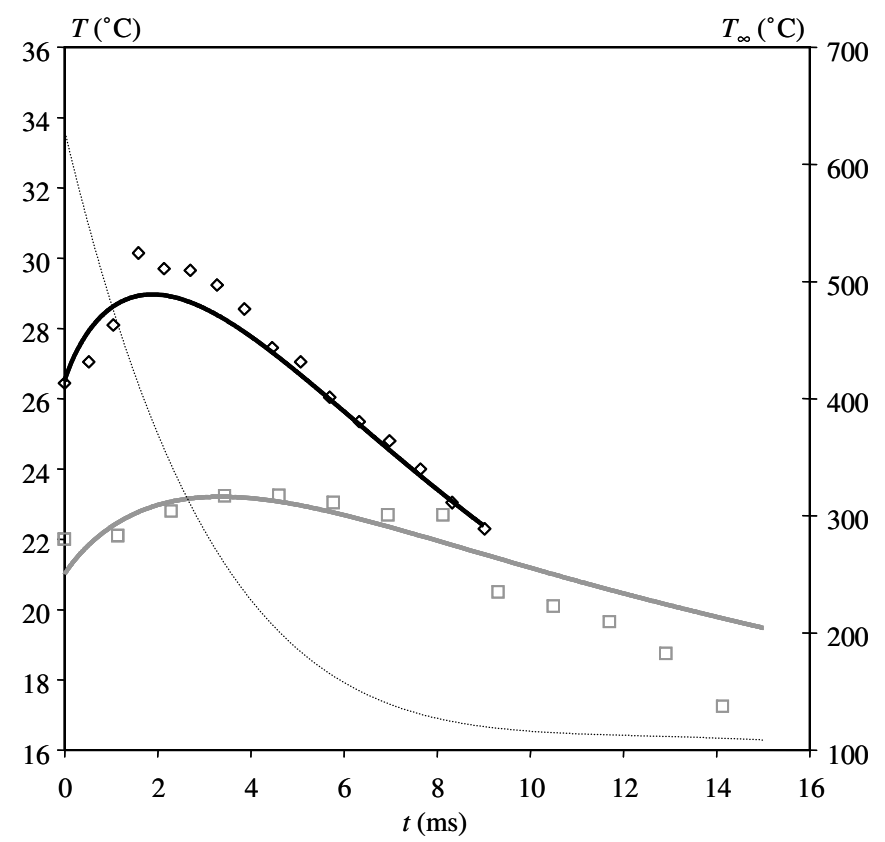

$$
\begin{array}{|llll|}
\hline \multicolumn{3}{|c|}{\text { Ambient temperature }} \\
D_{0}=133 \mu \mathrm{m} & D_{0}=227 \mu \mathrm{m} \\
\square \quad \begin{array}{lll}
\text { Experimental data } \\
\text { Numerical results }
\end{array} & \diamond & \text { Experimental data } \\
& & \text { Numerical results } \\
\hline
\end{array}
$$

Fig. 9. Temporal evolution of the droplet temperature for two droplet diameters and $Z_{0}=0.25$ in the hot air plume. Comparison to the numerical simulation results (origin of time at the droplet entrance in the hot air plume). purpose to mimic the Hill vortex model. Maps of the temperature and ethanol volume fraction calculated at $t=6.5 \mathrm{~ms}$ are presented in Fig. 4. These maps emphasize that the heat diffusion is much more efficient than the species diffusion. While acetone, the more volatile component, is quickly depleted at the droplet surface, it still remains at its initial fraction in large parts of the droplet core. The distribution of the volume fraction of ethanol follows the streamlines of the Hill vortices, which indicates that its transport is dominated by convection rather than by diffusion. The same behaviour is clearly not observed for the temperature field, since the mass Peclet number is about 50 times higher than the thermal Peclet number $\left(2 \times 10^{4}\right.$ against $\left.4 \times 10^{2}\right)$.

The assumption of a non-ideal liquid mixture was also considered in the tests. A noticeable effect can be observed in Fig. 3: the droplet temperature is reduced by almost $3.5^{\circ} \mathrm{C}$ when the non-ideal mixture model is considered. The non-ideality of the mixture promotes the vaporization of the liquid since the activity coefficients are greater than 1 whatever the ethanol fraction $(\gamma=1.2$ for ethanol when $Z=0.5$ ). Consequently, a more important amount of energy is removed to vaporize the liquid which could have been used to heat the droplet otherwise.

\subsection{Droplet evaporating at ambient temperature}

In this experiment, overheated droplets $\left(D_{0}=140 \mu \mathrm{m}\right.$, $T_{0}=45^{\circ} \mathrm{C}$ ) are injected into a quiescent atmosphere at ambient temperature. Initial compositions ranging from pure acetone to pure ethanol were tested. The initial conditions are summarized in Appendix in Table 1 and the evolutions of the droplet velocity
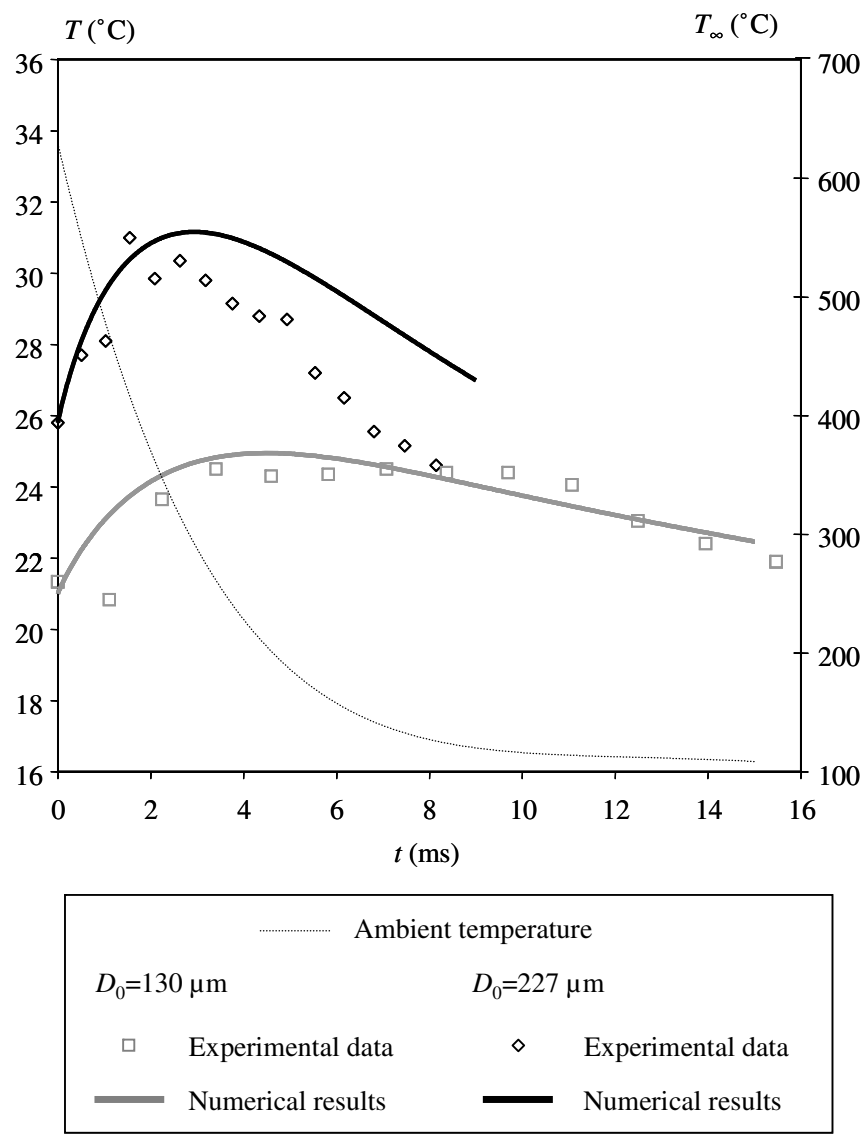

Fig. 10. Temporal evolution of the droplet temperature for two droplet diameters and $Z_{0}=0.5$ in the hot air plume. Comparison to the numerical simulation results (origin of time at the droplet entrance in the hot air plume). 
are given in the same table. The initial conditions are not exactly the same due to difficulties in obtaining a stable droplet stream for any investigated compositions. Computations are not started at the injector exit since the spherical droplets have not been formed yet. Moreover, the modelling of the heat and mass transfer is not relevant in the liquid disintegration phase.

For this reason, the initial condition in the simulation is generally taken $1 \mathrm{~ms}$ after the droplet injection.

In Fig. 5, temperature measurements at $t=6.5 \mathrm{~ms}$ are displayed for the investigated initial compositions, i.e. $Z_{0}=0,0.25,0.5,0.75$ and 1 . The cooling of the droplet is the highest for the smallest volume fraction of ethanol. The droplet temperature evolves from $16{ }^{\circ} \mathrm{C}$ for pure acetone to about $26^{\circ} \mathrm{C}$ for pure ethanol. An increase of the concentration of the less volatile component, i.e. ethanol, leads actually to a reduction of the vaporization rate and consecutively to an increase of the droplet temperature since less energy is consumed by vaporisation. Numerical simulations also reproduce this trend. However, it seems difficult to assert that considering the non-ideality of the acetone-ethanol mixture makes it possible to achieve lower discrepancies between the measurements and the simulations. Referring to the results of the non-ideal model, temperature is found to be lower at $Z_{0}=0.25$ than at $Z_{0}=0$, which is not the case of the experimental data, but in the same time it should be added that the differences could be explained by experimental errors as well.

To give a more complete comparison between the simulations and the measurements, temporal evolutions of the droplet temperature are displayed in Fig. $6\left(Z_{0}=0,0.5,1\right)$ and in Fig. $7\left(Z_{0}=0.25\right.$ and 0.75$)$. A generally good agreement between the calculated values and the experimental data can be noticed if one considered that the uncertainties of the measurements is about $1.3^{\circ} \mathrm{C}$. Once again, it is rather difficult to make a clear statement about the interest in accounting for the non-ideality. Effects resulting from a non-ideal mixing fall within the $1.3^{\circ} \mathrm{C}$ margin of the experimental uncertainties and therefore only balanced conclusions can be drawn. Consequently, only the ideal mixing will be considered in the further developments.

\subsection{Droplets evaporating in a hot air plume}

Droplets are injected in the hot air plume described in Section 2.1. For the sake of consistency, the same initial compositions were tested on droplets having very different initial sizes $\left(D_{0}=130\right.$ and $230 \mu \mathrm{m}$ ). The initial conditions and the evolutions of the droplet velocity corresponding to these configurations are summarized in Tables 2 and 3 in Appendix. Droplets are decelerated downstream due to the drag force and the gravity. For a given droplet size $\left(D_{0}=130 \mu \mathrm{m}\right.$ or $\left.D_{i}=230 \mu \mathrm{m}\right)$, the fuel was injected so that its temperature remains the same for any tested composition at the first measurement point chosen as the origin of time in the simulations. This point corresponds also to the droplet entrance into the hot air plume (Fig. 1).

Temporal evolutions of the droplet temperature are presented in Figs. 8-12 for the five initial compositions $Z_{0}=0,0.25,0.5$, 0.75 and 1 . For all the investigated initial compositions, except pure acetone, the droplets are heated up in the first stage of the
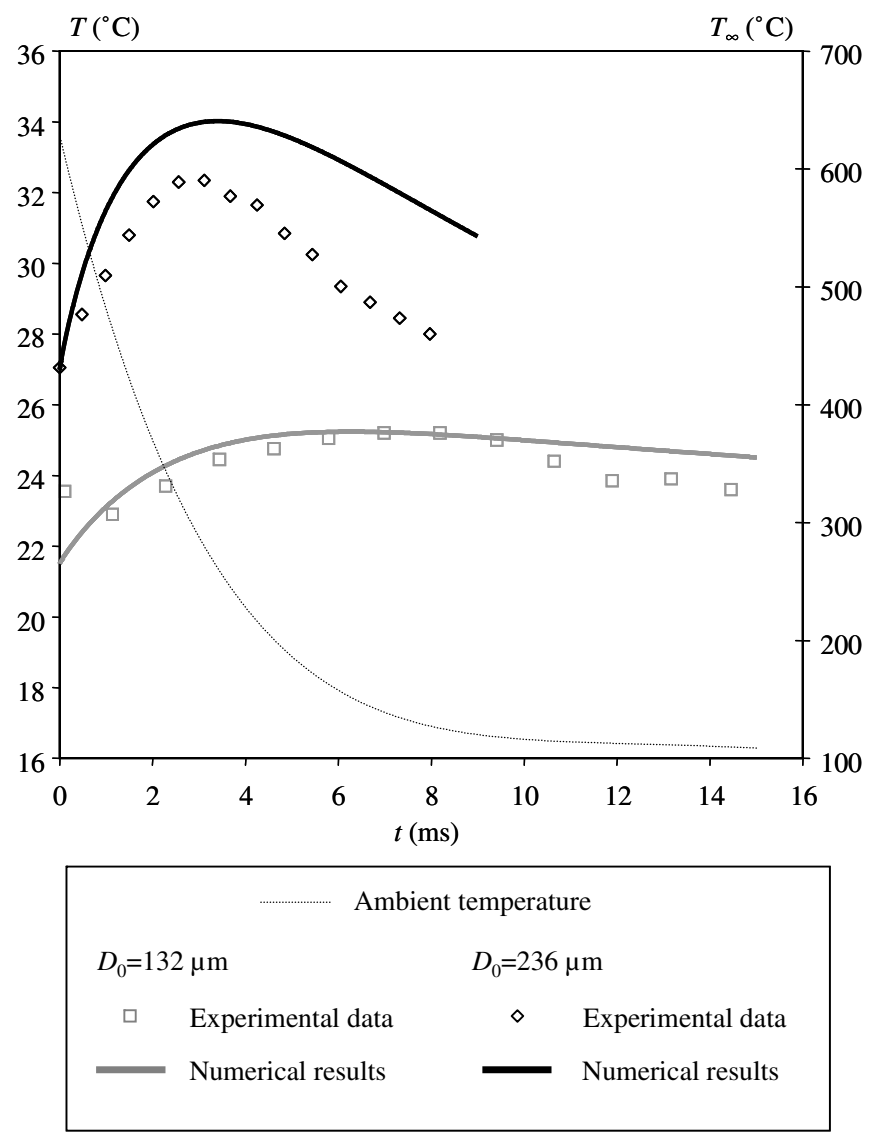

Fig. 11. Temporal evolution of the droplet temperature for two droplet diameters and $Z_{0}=0.75$ in the hot air plume. Comparison to the numerical simulation results (origin of time at the droplet entrance in the hot air plume).
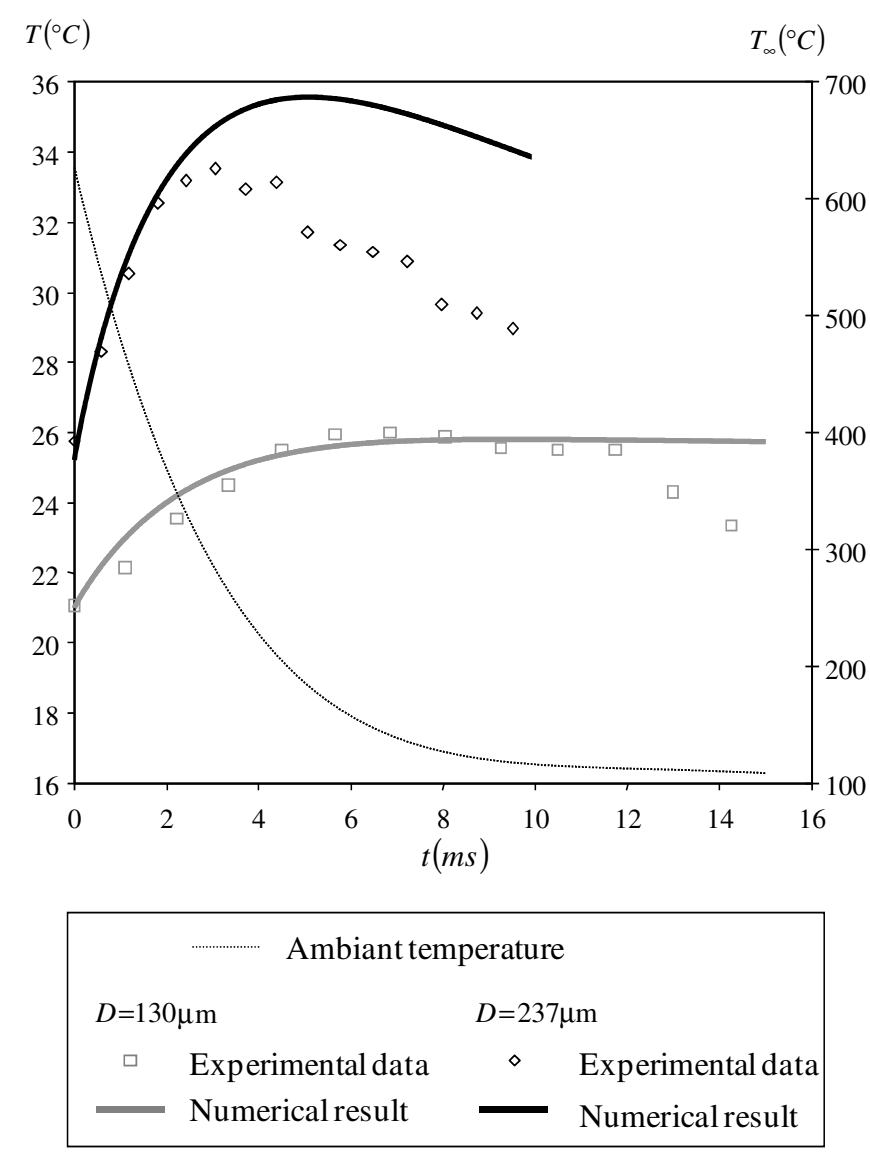

Fig. 12. Evolution droplet temperature for different droplet diameter and $Z=1$ in a hot air plume. Comparison to the numerical simulation results (origin of time at the droplet entrance in the hot air plume). 
evaporation, then the trend is inverting and the droplets temperature starts decreasing. Actually, when the air temperature decreases down to a certain limit, evaporation phenomena become dominant, i.e. evaporation uses more energy than the convective exchanges from the gaseous environment can provide leading to the cooling the droplet. This phenomenon takes place earlier for pure acetone droplets, since acetone is much more volatile than ethanol (the acetone saturated pressure is indeed about 3.7 times higher than the one of ethanol in the investigated range of temperature). As a consequence, the composition has a noticeable influence on the temperature maximum of the droplet heating as depicted in Fig. 13.

In the same figure, it can be seen that the simulations predict rather well the value of the temperature maximum for the lowest ethanol fractions. Nevertheless, the discrepancy is more noticeable in the case of the smallest droplets and for an ethanol volume fraction $Z_{0}=0.75$ or 1 . Figs. 11 and 12 illustrate how the temperature is overestimated by the simulation in these conditions.

As a summary, the agreement between the experiments and simulations is the better for the largest droplet size $\left(D_{0}=230 \mu \mathrm{m}\right)$. In Figs. 8-12, simulated curves and experimental data related to $D_{0}=230 \mu \mathrm{m}$ are indeed almost superimposed except at the end of the investigated time range when the measurements are not so reliable due to the loss of coherence of the droplet stream which tends to be destabilized after a certain time.

\subsection{Discussion of the results}

Attempt has been made to find out the origin of the differences between the experiments and the simulations. Since the turbulence is likely to accelerate heat and mass transfers at the droplet surface, it was interesting to determine whether the turbulence could have an effect on the results of the previously described

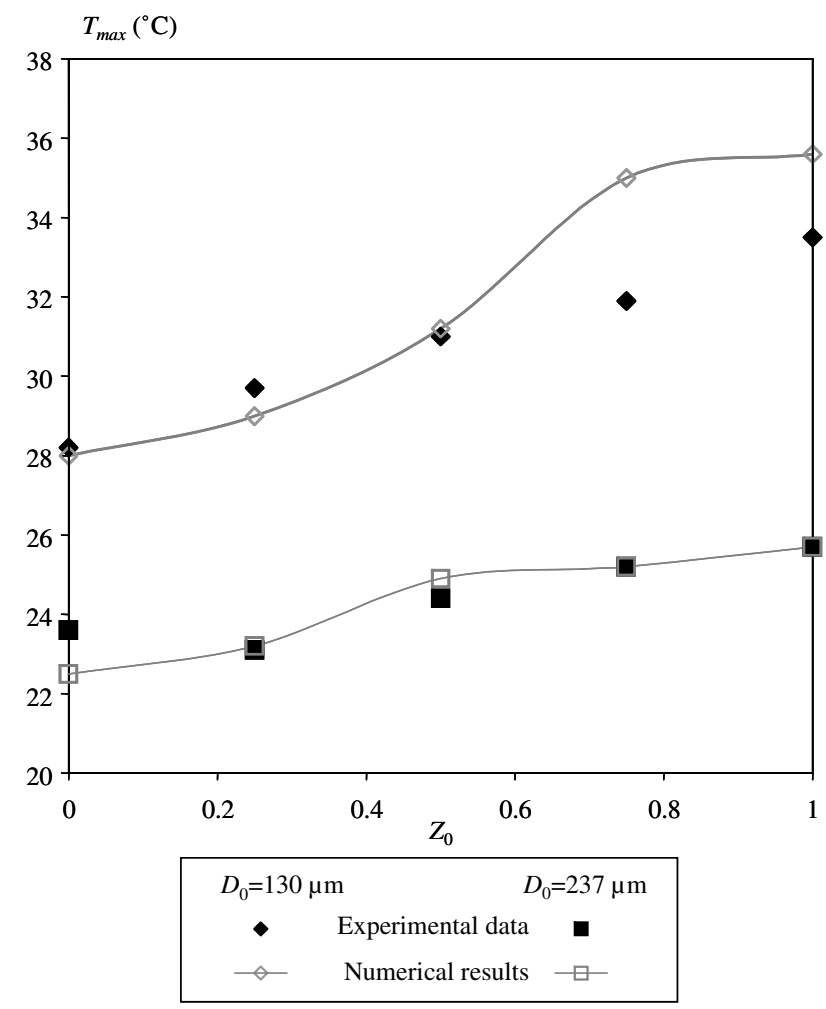

Fig. 13. Maximum droplet temperature as a function of the ethanol volume fraction for two droplet diameters. Comparison to the numerical simulation results (origin of time at the droplet entrance in the hot air plume). experiments. The distance $y_{\operatorname{tr}}$ where transition to turbulence occurs in the plume, can be evaluated by considering a critical Raleigh number of $10^{10}$ [24].

$y_{\mathrm{tr}}=\sqrt{\frac{\lambda R a_{\mathrm{c}}}{(\mathrm{g} \beta / a v) q}}$

In this expression, $\lambda, \beta, a, v$ and $R a_{\mathrm{c}}$ are, respectively, the air thermal conductivity, volumetric thermal expansion coefficient, heat diffusivity, kinematic viscosity and Rayleigh critical number. Taking into account that the power convected by the hot air plume is about $q=15 \mathrm{~W}$, numerical application of Eq. (22) locates approximately the transition to turbulence at $8 \mathrm{~cm}$ from the hot air inlet. This distance corresponds to the end of the exploration zone even for the biggest droplets. Consequently, the air flow surrounding the droplets is laminar and turbulence is not expected to play a significant role.

Results presented in Sections 4.2 and 4.3 reveal that the differences between the experiments and the simulations are the more important when both the fraction of the less volatile component and the air temperature are high. The attention was therefore focused mainly on the convective flux and more precisely on the Nusselt number. Considerations around the evaporation flux appears less relevant, since a rapid comparison between the vaporization and the convective heat fluxes shows that the vaporization heat flux is about 3-4 times smaller than the convective heat flux in the hot air plume (see Fig. 14). To study the consequence of an erroneous Nusselt number, a perturbation of $10 \%$ was applied to this number in the numerical simulation while keeping the Sherwood number unchanged. As observed in Fig. 15, the effect of the perturbation on the droplet temperature is the most important for the smallest droplets $\left(D_{0}=130 \mu \mathrm{m}\right)$ placed in the hot air flow. For the largest droplets $\left(D_{0}=230 \mu \mathrm{m}\right)$, the variation of the temperature is softened due the higher thermal inertia of these droplets. Effects of the perturbation can be also calculated for different ethanol volume fractions. The results of these computations are presented in Fig. 16 for $t=6 \mathrm{~ms}$. They confirm

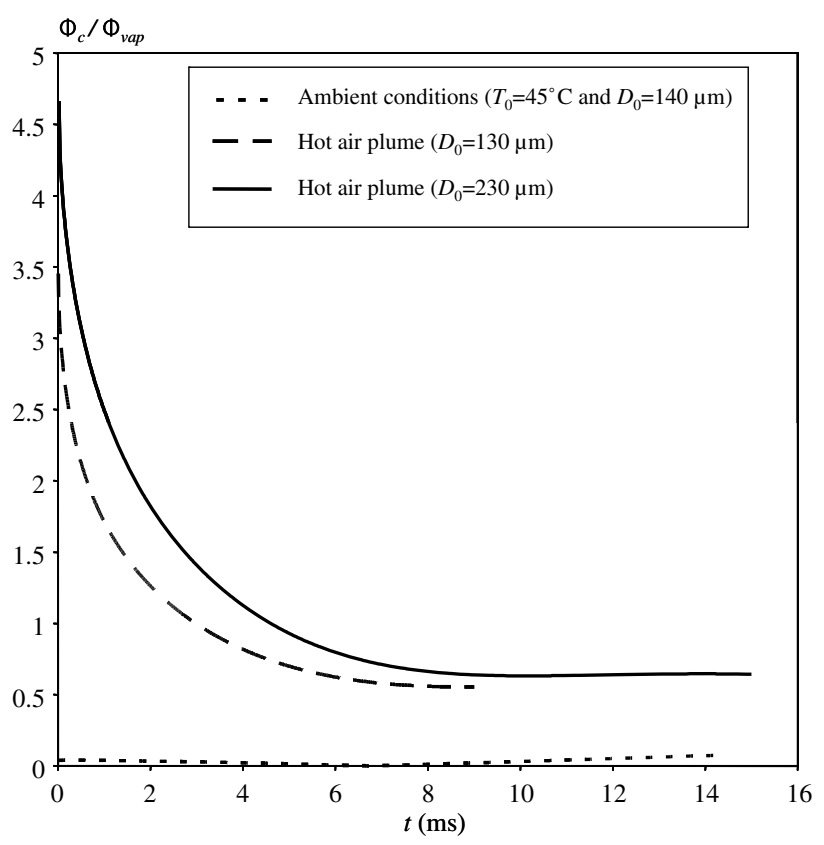

Fig. 14. Calculated temporal evolution of the ratio between the convective and evaporation heat fluxes for three configurations $\left(Z_{0}=0.5\right)$. 


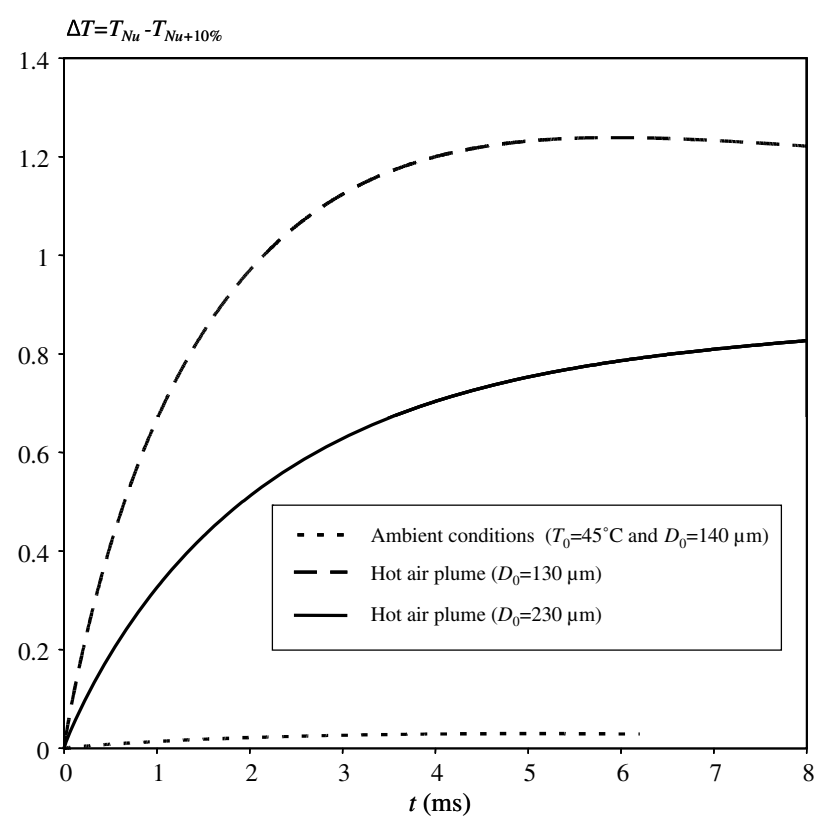

Fig. 15. Effect on the droplet temperature of a perturbation of $+10 \%$ of the Nusselt number as a function of time for three configurations $\left(Z_{0}=0.5\right)$.

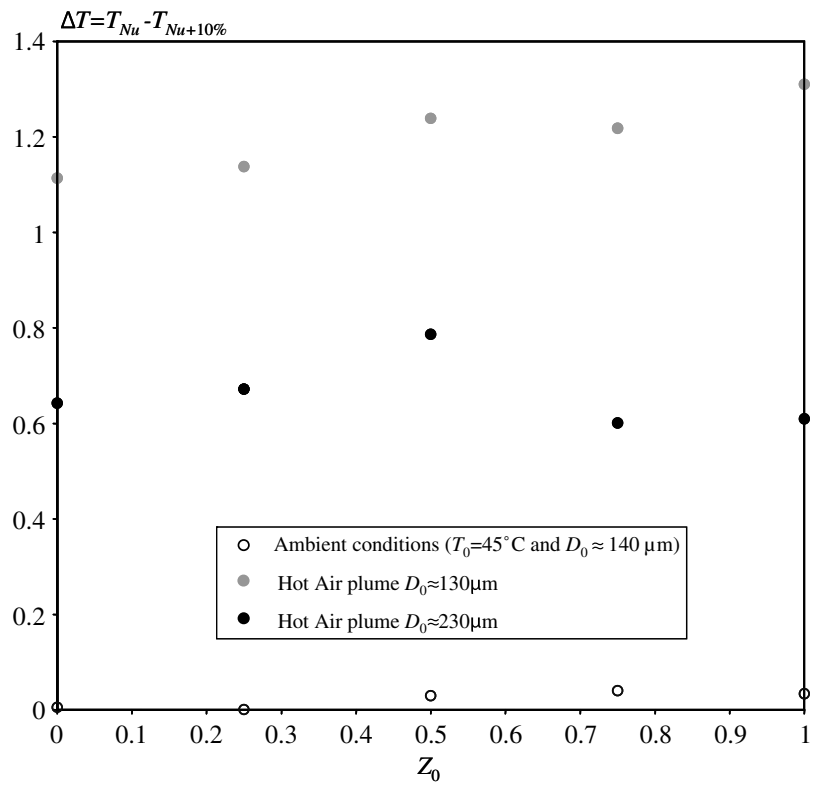

Fig. 16. Effect on the droplet temperature of a perturbation of $+10 \%$ of the Nusselt number as a function of the ethanol volume fraction for three configurations.

that the prediction of the simulations are more sensitive to an erroneous value of Nusselt number when dealing with the smallest droplets $\left(D_{0}=130 \mu \mathrm{m}\right)$ in the hot air plume. Having said this, the assumption of such an error appears rather likely. At this stage, Eqs. (16) and (17) can be questioned because of their central position in the estimation of the Nusselt and Sherwood numbers. These equations are used to describe how the interactions between the droplets are reducing the heat and mass transfers in comparison to the case of the isolated droplet. The modelling of the interaction effects is based on the idea that the Sherwood and Nusselt numbers are modified in the same extent by the droplet interactions whatever the conditions. This assumption seems rather strong and the proposed expression of $\eta$ (Eq. (17)) may be appropriate for a limited range of external conditions as well. Thermal variations of the physical properties of the gas surrounding the droplet may also have an effect on the interaction process. For example, diffusivities in the gas phase are central; they control the thickness of the boundary layers (thermal and mass) around the droplet. Depending on its efficiency, the diffusion is likely to affect the initial conditions of the scalar transport by the air flow and ultimately the distribution of this scalar (temperature or vapour concentration) around the droplets downstream. For example, diffusion coefficients are changing significantly with the ambient temperature. Presently the thermal and mass diffusivities are divided by 3 when the temperature evolves from 20 to $500^{\circ} \mathrm{C}$ in the hot air plume(for ethanol $D_{\text {eth }}=7.10^{-5} \mathrm{~m}^{2} / \mathrm{s}$ at $20^{\circ} \mathrm{C}$ and $2.5 .10^{-5} \mathrm{~m}^{2} / \mathrm{s}$ at $500{ }^{\circ} \mathrm{C}$, while $a_{\text {eth }}$ varies from $9.5 \times 10^{-5}$ to $3.1 \times 10^{-5} \mathrm{~m}^{2} / \mathrm{s}$ over the same temperature range).

\section{Conclusions}

The temperature of bi-component droplets have been measured under different aerothermal conditions. The case of overheated droplets evaporating at ambient temperature was first considered. The measurements were performed on droplets injected into an air plume at mid-range temperature (from 600 to $100{ }^{\circ} \mathrm{C}$ ). The accuracy of the measurement is estimated at about $1.3^{\circ} \mathrm{C}$. The experimental data were compared to a model based on the discrete components approach. Empirical correlations were used to account for the droplet to droplet interactions. Nusselt and Sherwood numbers are then reduced by an identical correction factor that depends on the reduced spacing parameter. Good agreements between the experimental data and the simulations are observed when droplets are evaporating near the ambient temperature or in the case of large droplets $(D \approx 230 \mu \mathrm{m})$ in the hot air plume. However, an improper evaluation of the heat fluxes is suspected in the other cases. In particular, the use of a correction factor, having the same value for the Sherwood and Nusselt numbers, appears questionable, since the droplet interactions are likely to be modified by the respective thickness of the mass and thermal boundaries layers as well as many physical properties of the gas phase. Future work will therefore focus on studying the heating and the evaporation of droplets in prescribed conditions that cover a wide range of physical properties.

\section{Acknowledgement}

This program has been conducted in the framework of the ASTRA program, supported by CNRS and ONERA.

\section{Appendix}

See Appendix Tables 1-3

Table 1

Conditions of the droplet injection at ambient temperature

\begin{tabular}{llll}
\hline$Z(\%)$ & Droplet diameter $(\mu \mathrm{m})$ & Distance parameter $(C)$ & Droplet velocity $(\mathrm{m} / \mathrm{s})$ \\
\hline 0 & 143 & 7.7 & $-0.316 t+12.81$ \\
25 & 134 & 8.7 & $-0.37 t+12.75$ \\
50 & 143 & 7.4 & $-0.448 t+12.71$ \\
75 & 137 & 7.5 & $-0.306 t+12.28$ \\
100 & 141 & 7.1 & $-0.344 t+12.30$ \\
\hline
\end{tabular}

Evolution of the droplet velocity as a function of the time elapsed in milliseconds from the injector exit. 
Table 2

Conditions of the droplet injection in the hot air plume

\begin{tabular}{llll}
\hline$Z(\%)$ & Droplet diameter $(\mu \mathrm{m})$ & Distance parameter $(C)$ & Droplet velocity $(\mathrm{m} / \mathrm{s})$ \\
\hline 0 & 126 & 7.6 & $-0.261 t+9.85$ \\
25 & 133 & 8.2 & $-0.285 t+9.59$ \\
50 & 130 & 7.3 & $-0.327 t+9.912$ \\
75 & 132 & 8 & $-0.353 t+10.2$ \\
100 & 130 & 6.7 & $-0.248 t+8.57$ \\
\hline
\end{tabular}

Evolution of the droplet velocity as a function of the time elapsed in milliseconds (origin of time is the injection point in the hot air plume).

Table 3

Conditions of the droplet injection in the hot air plume

\begin{tabular}{llll}
\hline$Z(\%)$ & Droplet diameter $(\mu \mathrm{m})$ & Distance parameter $(C)$ & Droplet velocity $(\mathrm{m} / \mathrm{s})$ \\
\hline 0 & 232 & 3.5 & $-0.056 t+8.58$ \\
25 & 227 & 3.5 & $-0.042 t+8.8$ \\
50 & 227 & 3.8 & $-0.043 t+8.9$ \\
75 & 236 & 3.7 & $-0.075 t+8.84$ \\
100 & 237 & 4 & $-0.096 t+9.07$ \\
\hline
\end{tabular}

Evolution of the droplet velocity as a function of the time elapsed in milliseconds (origin of time is the injection point in the hot air plume).

\section{References}

[1] Sirignano WA. Fuel droplets vaporization and spray combustion theory. Prog Energy Combust Sci 1983;9:291-322.

[2] Landis RB, Mills AF. Effect of internal resistance on the vaporization of binary droplets. In: Fifth int heat transfer conf Tokyo, Japan, 1974; Paper B7-9.

[3] Burger M, Schmehl R, Prommerberger K, Schäfer O, Koch R, Wittig S. Droplet evaporation modelling by the distillation curve model: accounting for kerozene fuel at elevated pressures. Int J Heat Mass Transfer 2003;46:4403-12.

[4] Homann S, Renz U. Numerical simulation of fuel sprays at high ambient pressure: the influence of real gas effects and gas solubility on droplet vaporisation. Int J Heat Mass Transfer 2003;46:3017-28.

[5] Kneer R, Scneider M, Noll B, Wittig S. Diffusion controlled evaporation of a multicomponent droplet: theoretical studies on the importance of variable liquid properties. Int J Heat Mass Transfer 1993;36:2403-15.

[6] Prakash S, Sirignano WA. Liquid fuel droplet heating with internal circulation. Int J Heat Mass Transfer 1978;21:885-95.
[7] Prakash S, Sirignano WA. Theory of convective fuel droplet vaporization with unsteady heat transfer in the circulating liquid phase. Int J Heat Mass Transfer 1980;23:253-68.

[8] Tong AY, Sirignano WA. Multicomponent droplet vaporization in high temperature gas. Combust Flame 1986;66:221-35.

[9] Abramzon B, Sirignano WA. Droplet vaporization model for spray combustion calculations. Int J Heat Mass Transfer 1989;32:1605-18.

[10] Le Clercq P, Bellan J. Direct numerical simulation of a transitional temporal mixing layer laden with multicomponent-fuel evaporating drop using continuous thermodynamics. Phys Fluids 2004:1884-907.

[11] Burger M, Schmehl R, Prommerberger K, Schäfer O, Koch R, Wittig S. Droplet evaporation modelling by the distillation curve model: accounting for kerozene fuel at elevated pressures. Int J Heat Mass Transfer 2003;46: 4403-12.

[12] Maqua C, Castanet G, Doué N, Lavergne G, Lemoine F. Temperature measurements of binary droplets using three color laser-induced fluorescence. Exp Fluids 2006;40:786-97.

[13] Lavieille P, Lemoine F, Lebouché M, Lavergne G. Evaporating and combusting droplet temperature measurement using two colors laser induced fluorescence. Exp Fluids 2001;31:45-55.

[14] Lavieille P, Lemoine F, Lebouché $M$. Investigation on temperature of evaporating droplets in linear stream using two-color laser-induced fluorescence. Combust Sci Technol 2002;174:117-42.

[15] Sangiovanni JJ, Labowsky M. Burning times of linear droplet arrays: a comparison of experiment and theory. Combust Flame 1982;47:15-30.

[16] Castanet G, Lavieille P, Lemoine F, Lebouché M, Atthasit A, Biscos Y, et al. Energetic budget on an evaporating monodisperse droplet stream using combined optical methods : evaluation of the convective heat transfer. Int $\mathrm{J}$ Heat Mass Transfer 2002;45:5053-67.

[17] Sirignano WA. Fluid dynamics and transport of droplets and sprays. Cambridge: University Press; 1999.

[18] Clift R, Grace JR, Weber ME. Bubbles, drops and particles. New York: Academic; 1978.

[19] Hubbard GL, Denny VE, Mills AF. Droplet evaporation: effects of transients and variable properties. Int J Heat Mass Transfer 1975;18:1003-8.

[20] Campbell SW, Wilseak RA, Thodos G. Vapor-liquid equilibrium measurements for the ethanol-acetone system at 372.7, 397.7 and 422.6 K. J Chem Eng Data 1987;32:357-62.

[21] Castanet G, Lebouché M, Lemoine F. Heat and mass transfer of combusting monodisperse droplets in linear stream. Int J Heat Mass Transfer 2005; 48:3261-75

[22] Atthasit A, Doué N, Biscos Y, Lavergne G. Influence of droplet concentration on the dynamics and evaporation of a monodisperse stream of droplets in evaporation regime. In: First international symposium on combustion and atmospheric pollution, St-Petersburg, Russia, July 8-11; 2003.

[23] Castanet G, Lavieille P, Lebouché M, Lemoine F. Experimental and theoretica investigation of the heating of combusting droplets in a linear stream. Combustion Sci Technol 2005;177:2395-422.

[24] Bejan A. Convection heat transfer. 2nd ed. New York: John Wiley and Sons Inc.; 1995. 